\section{Case Reports in Gastroenterology}

Case Rep Gastroenterol 2018;12:46-55

DOI: $10.1159 / 000486443$

Published online: February 6, 2018 (c) 2018 The Author(s)

Published by S. Karger AG, Basel www.karger.com/crg

This article is licensed under the Creative Commons Attribution-NonCommercial 4.0 International License (CC BY-NC) (http://www.karger.com/Services/OpenAccessLicense). Usage and distribution for commercial purposes requires written permission.

\title{
A Rare Multifocal Pattern of Type 2 Autoimmune Pancreatitis with Negative IgG4: A Potential Diagnostic Pitfall That May Mimic Multifocal Pancreatic Adenocarcinoma
}

\author{
Partha Hota $^{a} \quad$ Tejas Patel $^{b} \quad$ Xiaofeng Zhao $^{a} \quad$ Nirag Jhala $^{a}$ \\ Omar Agosto ${ }^{a}$ \\ aDepartment of Radiology, Temple University Hospital, Philadelphia, PA, USA; \\ ${ }^{\mathrm{b}}$ Atlantic Medical Imaging, Galloway, NJ, USA
}

\section{Keywords}

Autoimmune pancreatitis · Negative IgG4 · Pancreatic mass

\begin{abstract}
Autoimmune pancreatitis (AIP) is an increasingly recognized form of acute pancreatitis characterized by obstructive jaundice with a rapid and dramatic treatment response to steroid therapy. Recently, AIP has been divided into two distinct phenotypes: lymphoplasmocytic sclerosing pancreatitis AIP (type 1) and idiopathic duct-centric pancreatitis AIP (type 2); each of which have their own distinct demographics, diagnostic criteria, and histopathological features. We report, to the best of our knowledge, the first case of a multifocal pattern of type 2 AIP characterized with both CT and MR imaging. This rare imaging pattern of AIP may mimic the appearance of more worrisome malignant etiologies such as multifocal pancreatic adenocarcinoma or lymphoma, with overlapping imaging characteristics potentially com-
\end{abstract}




\section{Case Reports in Gastroenterology}

Case Rep Gastroenterol 2018;12:46-55

DOI: $10.1159 / 000486443$

(c) 2018 The Author(s). Published by S. Karger AG, Base www.karger.com/crg

Hota et al.: A Rare Multifocal Pattern of Type 2 AIP with Negative IgG4: A Potential

Diagnostic Pitfall That May Mimic Multifocal Pancreatic Adenocarcinoma

plicating or delaying diagnosis. Therefore, recognition of this atypical pattern of AIP and avoidance of this potential diagnostic pitfall is crucial.

(C) 2018 The Author(s)

Published by S. Karger AG, Basel

\section{Introduction}

Autoimmune pancreatitis (AIP) is an increasingly recognized form of acute pancreatitis characterized by obstructive jaundice with a rapid and dramatic treatment response to steroid therapy. First described in 1991 by Kawaguchi et al. [1] as lymphoplasmocytic sclerosing pancreatitis (LPSP) and subsequently linked to an autoimmune mechanism in 1995 by Yoshida et al. [2], the concept of AIP has since been widely recognized. In 2001, Hamano et al. [3] demonstrated a relationship between elevated serum immunoglobulin-4 (IgG4) levels and LPSP AIP; however, only recently has AIP been divided into two distinct phenotypes: LPSP AIP (type 1) and idiopathic duct-centric pancreatitis AIP (type 2); each type has its own distinct demographics, diagnostic criteria, and histopathological features [4, 5]. Crosssectional imaging with CT and MR imaging is crucial in the diagnosis of both AIP subtypes with several imaging patterns having been described [6]. Of these imaging patterns, a rare multifocal pattern of AIP has only been reported 9 times in the literature, with all previous reported cases of multifocal AIP having been associated with elevated serum IgG4 levels suggesting the type 1 phenotype [7]. We herein report, to the best of our knowledge, the first case of a multifocal pattern of type 2 AIP characterized with both CT and MR imaging. This rare imaging pattern of AIP may mimic the appearance of more worrisome malignant etiologies such as multifocal pancreatic adenocarcinoma or lymphoma, with overlapping imaging characteristics potentially complicating or delaying diagnosis. Therefore, recognition of this atypical pattern of AIP and avoidance of this potential diagnostic pitfall is crucial.

\section{Case Report}

A 53-year-old African-American female with a history of gastric ulcer was admitted to our hospital with a 2-week history of abdominal pain with nausea and a 2-month history of an unexplained 20-pound weight loss. Physical examination demonstrated right upper quadrant abdominal tenderness with radiation to the back. Vital signs were within normal limits without tachycardia or fever. Laboratory data on admission (Table 1) demonstrated an elevated total bilirubin, direct bilirubin, alkaline phosphatase, alanine aminotransferase, and aspartate aminotransferase. Serum lipase was within normal limits measuring $48 \mathrm{U} / \mathrm{L}$. The remainder of the laboratory data was otherwise within normal limits.

Contrast-enhanced CT imaging demonstrated slight diffuse enlargement of the pancreas with heterogeneous attenuation and the suggestion of multifocal heterogeneous hypoattenuating hypoenhancing masses with mild segmental pancreatic ductal narrowing and minimal ductal dilatation (Fig. 1a-c). Contrast-enhanced MR imaging on a 1.5-T scanner confirmed these findings (Fig. 1d-i) with areas of mild main pancreatic ductal narrowing also confirmed on magnetic resonance cholangiopancreatography (MRCP) (Fig. 1j). Subsequent endoscopic ultrasound with fine needle aspiration of the mass-like lesions was performed. In 


\section{Case Reports in \\ Gastroenterology}

Case Rep Gastroenterol 2018;12:46-55

DOI: $10.1159 / 000486443$

(C) 2018 The Author(s). Published by S. Karger AG, Base www.karger.com/crg

Hota et al:: A Rare Multifocal Pattern of Type 2 AIP with Negative IgG4: A Potential

Diagnostic Pitfall That May Mimic Multifocal Pancreatic Adenocarcinoma

addition, endoscopic retrograde cholangiopancreatography (ERCP) was performed which demonstrated a focal stricture in the common bile duct that was subsequently stented. $\mathrm{Pa}-$ thology from the fine needle aspiration demonstrated areas of fibrosis and periductal lymphoplasmocytic infiltrate as well as neutrophils in close approximation to the ductal epithelium (Fig. 2). No evidence of malignancy or IgG4-positive plasma cells was identified. Given the presence of fibrosis and lack of malignancy, AIP was suspected. Subsequent serum total immunoglobulin (IgG) was within normal limits, and serum IgG4 was only marginally elevated measuring $90 \mathrm{mg} / \mathrm{dL}$ (normal range: $4-86 \mathrm{mg} / \mathrm{dL}$ ).

The patient was empirically treated for AIP with oral steroids and her symptoms and liver function tests markedly improved during the course of her hospital stay. The patient was discharged on oral steroid therapy and returned for surveillance imaging 1 month later. Contrast-enhanced CT imaging at that time demonstrated interval resolution of the previously seen multifocal pancreatic masses and areas of extrinsic compression of the main pancreatic duct (Fig. 3). Given the rapid treatment response to steroid therapy, absence of IgG4positive plasma cells on pathology, and lack of extrapancreatic manifestations of IgG4related disease, the unifying final diagnosis was a multifocal pattern of type 2 AIP.

\section{Discussion}

AIP is a rare form of chronic pancreatitis, with several studies reporting an approximate incidence of up to $6 \%$ in patients with chronic pancreatitis [6, 8-11]. Originally favored to solely reflect the pancreatic manifestation of IgG4-related disease, only recently has AIP been divided into two distinct phenotypes: type 1 and type 2; each with specific demographics, disease associations, and histopathological features (Table 2) [3-5].

Type 1 AIP, also known as LPSP AIP, is the pancreatic manifestation of IgG4-related systemic disease and is characterized by diffuse enlargement of the pancreas, elevated serum IgG4 levels, and pancreatic infiltration with IgG4-positive plasma cells [6, 12]. Demographically, type 1 AIP has a higher incidence in older males and those from Asia [12]. Coexisting extrapancreatic pathologies are common and include Sjögren-like xerostomia and xerophthalmia, hilar lymphadenopathy, sclerosing cholangitis, interstitial nephritis, and retroperitoneal fibrosis $[12,13]$. In addition, disease associations such as lymphocytic hypophysitis, orbital pseudotumor, chronic thyroiditis, gallbladder and gastric involvement, and prostatitis have been reported $[6,12]$. Although type 2 AIP, also known as idiopathic duct-centric pancreatitis AIP or AIP with granulocytic epithelial lesions (GELs), also demonstrates diffuse pancreatic enlargement, the presence of ductal epithelial granulocytic infiltration with or without destruction and obliteration of the pancreatic duct as well as the absence or paucity of IgG4-positive plasma cells are key differentiating factors from type 1 AIP $[6,12,14]$. Type 2 AIP also tends to affect a different patient demographic, favoring younger patients in the United States and Europe over Asia without a clear gender predilection [12, 15]. Unlike type 1 AIP, extrapancreatic manifestations are uncommon in type 2 AIP, with the exception of inflammatory bowel disease, which has been cited in up to $30 \%$ of cases [12].

Clinical manifestations of both AIP subtypes are similar, and overlapping symptomatology can make clinical differentiation of both entities difficult. While patients with either phenotype may present with both obstructive jaundice and abdominal pain, type 1 AIP is more 


\section{Case Reports in Gastroenterology}

Case Rep Gastroenterol 2018;12:46-55

DOI: $10.1159 / 000486443$

(c) 2018 The Author(s). Published by S. Karger AG, Basel www.karger.com/crg

Hota et al:: A Rare Multifocal Pattern of Type 2 AIP with Negative IgG4: A Potential

Diagnostic Pitfall That May Mimic Multifocal Pancreatic Adenocarcinoma

closely associated with the former and type 2 AIP with the latter [12]. Additional nonspecific symptoms present in both subtypes include jaundice and unintentional weight loss that may mimic those of pancreatic malignancy $[13,16]$. Misdiagnosis based on imaging may lead to inappropriate treatment with at least two studies describing AIP found in approximately 2$6 \%$ of patients who had undergone pancreatic resection for suspected adenocarcinoma $[9$, 10]. Therefore, imaging has a crucial role in differentiating AIP from other pancreatic pathologies, with histopathology playing a paramount part in differentiating between AIP subtypes.

Both AIP subtypes on quadriphasic CT imaging are indistinguishable as these entities are morphologically similar. Three recognized imaging patterns of AIP have been described: diffuse pancreatic enlargement, focal or segmental lesions, and multifocal lesions. The most common form of AIP presents as a diffusely enlarged sausage-shaped hypoattenuating pancreas demonstrating decreased enhancement on arterial phase imaging and delayed enhancement on equilibrium phase imaging [17]. Additionally, a contiguous peripheral capsule-like rim of hypoattenuation with loss of the normal fatty lobulations has been reported in $14-48 \%$ of cases $[17,18]$. The focal or segmental form of AIP typically involves the pancreatic head and is the second-most common form [6,17]. Even rarer, as in our case, is the multifocal form that has only been described 9 times in the literature and associated, until now, with only type 1 AIP [7]. Compared to the normal pancreatic parenchyma, focal and multifocal lesions may be iso- or hypoattenuating and may mimic malignant etiologies such as pancreatic adenocarcinoma or pancreatic lymphoma $[6,18,19]$.

Similar to CT, the MRI appearance of both AIP subtypes are indistinguishable, with affected portions of the pancreatic parenchyma displaying T1 hypointensity and inhomogeneous T2 slight hyperintensity. Postcontrast imaging findings are similar to CT with lesions demonstrating hypoenhancement on arterial phase imaging and delayed enhancement on equilibrium phase imaging. The well-demarcated capsule-like rim of hypoattenuation on CT imaging demonstrates intrinsic T1 and T2 hypointensity and moderate post-contrast enhancement [6]. MRI is superior to CT in differentiating AIP from other pancreatic malignancies. Echoplanar water diffusion-weighted MRI (DWI) and extrapolated apparent diffusion coefficient maps (ADC) have been reported as a potential pulse sequence to help differentiate AIP from pancreatic adenocarcinoma [6]. AIP tends to have greater restricted water diffusion manifesting as areas of higher DWI signal intensity and lower ADC signal intensity compared to pancreatic adenocarcinoma and normal pancreatic parenchyma [6, 20-22]. At least two groups have reported ADC thresholds and averages between $0.88 \times 10^{-3}$ and 1.012 $\times 10^{-3} \mathrm{~mm}^{2} / \mathrm{s}$ on 1.5 -T scanners, favoring a diagnosis of AIP over pancreatic adenocarcinoma $[21,22]$. The T2 and post-contrast appearance of AIP have also been reported to be useful in differentiating multifocal AIP from multifocal pancreatic lymphoma, with lymphoma displaying more homogeneous T2 hyperintensity and lack of delayed enhancement on equilibrium phase imaging [19]. In our case, the mean ADC value for the lesions was $1.05 \times 10^{-3} \pm 0.1 \times$ $10^{-3} \mathrm{~mm}^{2} / \mathrm{s}$ with areas of iso to slight T2 hyperintensity favoring AIP as the underlying etiology rather than adenocarcinoma and lymphoma based on imaging alone.

MRCP is advantageous compared to CT imaging in evaluation of the main pancreatic duct. Segmental and multifocal narrowing of the pancreatic duct secondary to AIP lesions is common with narrowing usually longer than $3 \mathrm{~cm}$ in the diffuse form [6, 23]. Upstream ductal dilatation in AIP may be present on MRCP but is typically less pronounced than dilatation 


\section{Case Reports in Gastroenterology}

Case Rep Gastroenterol 2018;12:46-55

DOI: $10.1159 / 000486443$

(C) 2018 The Author(s). Published by S. Karger AG, Base www.karger.com/crg

Hota et al:: A Rare Multifocal Pattern of Type 2 AIP with Negative IgG4: A Potential

Diagnostic Pitfall That May Mimic Multifocal Pancreatic Adenocarcinoma

secondary to pancreatic adenocarcinoma as was seen in our case [6, 21, 23]. At least one study has suggested $4 \mathrm{~mm}$ as the preferred threshold to distinguish between AIP and pancreatic adenocarcinoma [6,21]. Dynamic MR imaging following secretin administration has also been reported as a potential tool in distinguishing benign strictures of AIP from malignant strictures of pancreatic adenocarcinoma with patency of the main pancreatic duct demonstrated through the mass-like lesions of AIP following secretin stimulation, the socalled "duct penetrating sign" [24, 25].

Ancillary findings on CT and MR imaging include milder than expected peripancreatic stranding reflecting phlegmon or post-inflammatory fibrosis, as was seen in our patient [6]. Additional reported findings of AIP are the presence of calcifications (14-32\%) and pseudocyst formation (10-12\%) that typically present in the subacute or chronic phases [26, 27]. While these are nonspecific findings, as they may be seen in the setting of entities such as alcohol induced chronic pancreatitis, and were also not demonstrated in our case, their presence should not exclude the diagnosis of AIP in the appropriate clinical scenario [17].

Although macroscopically both subtypes of AIP are indistinguishable, histology plays a pivotal role in their differentiation. Type 1 AIP is predominantly characterized by the presence of IgG4-positive plasma cells (>10 cells/HPF) $[5,16,28]$. Nonspecific findings of type 1 AIP include periductal lymphoplasmocytic infiltrate affecting medium-sized ducts and acini, periductal and perilobular storiform fibrosis, and vasculitis secondary to lymphoplasmocytic infiltrate leading to phlebitis and arteritis $[16,28]$. Type 2 AIP is characterized by the presence of GELs that are absent in type 1 AIP $[16,29]$. In addition, none or few $(<10$ cells/HPF) IgG4-positive plasma cells are present in contrast to type 1 AIP [16]. While lymphoplasmocytic infiltrate, storiform fibrosis, and vasculitis may be present in type 2 AIP, these findings are far less common in comparison to type 1 AIP [16]. In practice, adequate tissue sampling from ERCP of AIP lesions can be difficult, and differentiation between subtypes may rely on clinical features such as relapse following steroid treatment or the presence or absence of extrapancreatic manifestations [30]. In our patient, while periductal lymphoplasmocytic infiltrate and fibrosis were identified, the presence of neutrophils in close approximation to the ducts suggesting GEL presence, absence of IgG4 positive plasma cells, and lack of extrapancreatic manifestations of IgG4-related disease strongly favor a diagnosis of type 2 AIP.

Both subtypes of AIP have a dramatic clinical response following oral corticosteroid treatment with response to treatment monitored clinically by normalized liver function tests reflecting resolving biliary obstruction, and anatomically by imaging [6]. Posttreatment CT and MR imaging demonstrate decreased size of pancreatic lesions with isointensity and isoenhancement to that of the normal pancreatic parenchyma [6,31]. In the majority of cases, mild pancreatic ductal dilatation will return to normal reflecting resolved benign stricturing with persistent narrowing present in only a minority [31, 32]. Typically steroid treatment is tapered after 4 weeks in the presence of imaging and laboratory improvement [6]. In our case, within 1 month of the initiation of steroid therapy, the multifocal lesions of type 2 AIP were no longer visualized with normalized pancreatic attenuation and enhancement. Oral steroid therapy was consequently discontinued. Disease relapse following steroid treatment is uncommon in type 2 AIP in contrast to type 1 AIP where up to $40 \%$ of patients may experience an additional episode [33,34]. In severe cases that are nonresponsive to steroid therapy, surgical intervention with pancreaticoduodenectomy or distal pancreatectomy may be performed depending on the degree and location of pancreatic involvement [6, 
35]. In rare cases of diffuse AIP refractory to steroid treatment, total pancreatectomy has been reported [35].

In conclusion, this is the first reported case of a rare multifocal form of AIP with clinical and histopathological features heavily favoring the type 2 phenotype characterized on CT and MR imaging as well as histopathology. The atypical multifocal presentation and absence of IgG4-positive plasma cells can mimic multifocal pancreatic malignancy and may present as a potential diagnostic pitfall, possibly delay treatment, or lead to unnecessary surgery. Therefore, in such a setting where AIP is suspected, knowledge of the individual subtypes, an empiric trial of corticosteroid treatment, and short-term follow-up imaging to assess for temporal resolution, in addition to tissue diagnosis is crucial in making an accurate diagnosis.

\section{Statement of Ethics}

This article was written in compliance with our institutional ethical standards.

\section{Disclosure Statement}

The authors have no conflicts of interest to disclose.

\section{References}

1 Kawaguchi K, Koike M, Tsuruta K, et al: Lymphoplasmacytic sclerosing pancreatitis with cholangitis: a variant of primary sclerosing cholangitis extensively involving pancreas. Hum Pathol 1991;22:387395.

2 Yoshida K, Toki F, Takeuchi T, Watanabe S, Shiratori K, Hayashi N: Chronic pancreatitis caused by an autoimmune abnormality: proposal of the concept of autoimmune pancreatitis. Dig Dis Sci 1995;40: 1561-1568.

3 Hamano H, Kawa S, Horiuchi A, et al: High serum IgG4 concentrations in patients with sclerosing pancreatitis. N Engl J Med 2001;344:732-738. Chari ST, Kloeppel G, Zhang L, Notohara K, Lerch MM, Shimosegawa T: Histopathologic and clinical subtypes of autoimmune pancreatitis: the Honolulu consensus document. Pancreas 2010;39:549-554. Shimosegawa T, Chari ST, Frulloni L, Kamisawa T, Kawa S, Mino-Kenudson M, Kim MH, Klöppel G, Lerch MM, Löhr M, Notohara K, Okazaki K, Schneider A, Zhang L: International consensus diagnostic criteria for autoimmune pancreatitis: guidelines of the International Association of Pancreatology. Pancreas 2011;40:352-358.

6 Crosara S, D'Onofrio M, De Robertis R, et al: Autoimmune pancreatitis: multimodality non-invasive imaging diagnosis. World J Gastroenterol 2014;20:16881-16890.

-7 Suzumura K, Hatano E, Uyama N, Okada T, Asano Y, Hai S, Nakasho K, Fujimoto J: Multifocal mass lesions in autoimmune pancreatitis. Case Rep Gastroenterol 2017;11:678-685.

-8 Finkelberg DL, Sahani D, Deshpande V, Brugge WR: Autoimmune pancreatitis. N Engl J Med 2006;355:2670-2676.

-9 Yadav D, Notahara K, Smyrk TC, Clain JE, Pearson RK, Farnell MB, Chari ST: Idiopathic tumefactive chronic pancreatitis: clinical profile, histology, and natural history after resection. Clin Gastroenterol Hepatol 2003;1:129-135.

10 Smith CD, Behrns KE, van Heerden JA, Sarr MG: Radical pancreatoduodenectomy for misdiagnosed pancreatic mass. Br J Surg 1994;81:585-589. 


\section{Case Reports in Gastroenterology}

Case Rep Gastroenterol 2018;12:46-55

DOI: $10.1159 / 000486443$

(C) 2018 The Author(s). Published by S. Karger AG, Basel www.karger.com/crg

Hota et al:: A Rare Multifocal Pattern of Type 2 AIP with Negative IgG4: A Potential

Diagnostic Pitfall That May Mimic Multifocal Pancreatic Adenocarcinoma

$>11$

Nishimori I, Tamakoshi A, Kawa S, Tanaka S, Takeuchi K, Kamisawa T, Saisho H, Hirano K, Okamura K, Yanagawa N, Otsuki M: Influence of steroid therapy on the course of diabetes mellitus in patients with autoimmune pancreatitis: findings from a nationwide survey in Japan. Pancreas 2006;32:244-248. Okazaki K, Uchida K, Miyoshi H, Ikeura T, Takaoka M, Nishio A: Recent concepts of autoimmune pancreatitis and IgG4-related disease. Clin Rev Allergy Immunol 2011;41:126-138.

Okazaki K, Uchida K, Koyabu M, et al: Recent advances in the concept and diagnosis of autoimmune pancreatitis and IgG4-related disease. J Gastroenterol 2011;46:277.

Kamisawa T, Takuma K, Tabata T, Inaba Y, Egawa N, Tsuruta K, Hishima T, Sasaki T, Itoi T: Serum IgG4negative autoimmune pancreatitis. J Gastroenterol 2011;46:108-116.

Sugumar A, Kloppel G, Chari ST: Autoimmune pancreatitis: pathologic subtypes and their implications for its diagnosis. Am J Gastroenterol 2009;104:2308-2310.

Klöppel G: Type 2 autoimmune pancreatitis. Pancreapedia DOI: 10.3998/panc.2013.22.

Takahashi N: CT and MR features of autoimmune pancreatitis. Pancreapedia DOI: 10.3998/ panc.2013.15.

Suzuki K, Itoh S, Nagasaka T, Ogawa H, Ota T, Naganawa S: CT findings in autoimmune pancreatitis: assessment using multiphase contrast-enhanced multisection CT. Clin Radiol 2010;65:735-743. Ishigami K, et al: MRI findings of pancreatic lymphoma and autoimmune pancreatitis: a comparative study. Eur J Radiol 2010;74:e22-e28.

Ichikawa T, Erturk SM, Motosugi U, Sou H, Iino H, Araki T, Fujii H: High-b value diffusion-weighted MRI for detecting pancreatic adenocarcinoma: preliminary results. AJR Am J Roentgenol 2007;188:409-414. Muhi A, Ichikawa T, Motosugi U, Sou H, Sano K, Tsukamoto T, Fatima Z, Araki T: Mass-forming autoimmune pancreatitis and pancreatic carcinoma: differential diagnosis on the basis of computed tomography and magnetic resonance cholangiopancreatography, and diffusion-weighted imaging findings. J Magn Reson Imaging 2012;35:827-836.

Kamisawa T, Takuma K, Anjiki H, Egawa N, Hata T, Kurata M, Honda G, Tsuruta K, Suzuki M, Kamata N, Sasaki T: Differentiation of autoimmune pancreatitis from pancreatic cancer by diffusion-weighted MRI. Am J Gastroenterol 2010;105:1870-1875.

Kamisawa T, Tu Y, Egawa N, Tsuruta K, Okamoto A, Kodama M, Kamata N: Can MRCP replace ERCP or the diagnosis of autoimmune pancreatitis? Abdom Imaging 2009;34:381-384.

Carbognin G, Girardi V, Biasiutti C, Camera L, Manfredi R, Frulloni L, Hermans JJ, Mucelli RP: Autoimmune pancreatitis: imaging findings on contrast-enhanced MR, MRCP and dynamic secretinenhanced MRCP. Radiol Med 2009;114:1214-1231.

Ichikawa T, Sou H, Araki T, Arbab AS, Yoshikawa T, Ishigame K, Haradome H, Hachiya J: Ductpenetrating sign at MRCP: usefulness for differentiating inflammatory pancreatic mass from pancreatic carcinomas. Radiology 2001;221:107-116.

Suzuki K, Itoh S, Nagasaka T, Ogawa H, Ota T, and Naganawa S: CT findings in autoimmune pancreatitis: assessment using multiphase contrast-enhanced multisection CT. Clin Radiol 2010;65:735-743.

Takahashi N, Fletcher JG, Fidler JL, Hough DM, Kawashima A, Chari ST: Dual-phase CT of autoimmune pancreatitis: a multireader study. AJR Am J Roentgenol 2008;190:280-286.

Notohara K, Zhang L: Histology of autoimmune pancreatitis. Pancreapedia DOI: 10.3998/panc.2013.12.

Zamboni G, Lüttges J, Capelli P, Frulloni L, Cavallini G, Pederzoli P, Leins A, Longnecker D, Klöppel G: Histopathological features of diagnostic and clinical relevance in autoimmune pancreatitis: a study on 53 resection specimens and 9 biopsy specimens. Virchows Arch 2004;445:552-563.

Draganov PV, et al: Diagnostic accuracy of conventional and cholangioscopy-guided sampling of indeterminate biliary lesions at the time of ERCP: a prospective, long-term follow-up study. Gastrointest Endosc 2012;75:347-353.

Manfredi R, Graziani R, Cicero C, Frulloni L, Carbognin G, Mantovani W, Mucelli RP: Autoimmune pancreatitis: CT patterns and their changes after steroid treatment. Radiology 2008;247:435-443. Manfredi R, Frulloni L, Mantovani W, Bonatti M, Graziani R, Pozzi Mucelli R: Autoimmune pancreatitis: pancreatic and extrapancreatic MR imaging-MR cholangiopancreatography findings at diagnosis, after steroid therapy, and at recurrence. Radiology 2011;260:428-436.

Gardner TB, Chari ST: Autoimmune pancreatitis. Gastroenterol Clin North Am 2008;37:439-460.

Chari ST, Murray JA: Autoimmune pancreatitis, part II: the relapse. Gastroenterology 2008;134: 625-628.

Kamisawa T, Satake K: Clinical management of autoimmune pancreatitis. Adv Med Sci 2007;52:61-65. 


\section{Case Reports in \\ Gastroenterology}

Case Rep Gastroenterol 2018;12:46-55

DOI: $10.1159 / 000486443$

(C) 2018 The Author(s). Published by S. Karger AG, Basel www.karger.com/crg

Hota et al: A Rare Multifocal Pattern of Type 2 AIP with Negative IgG4: A Potential

Diagnostic Pitfall That May Mimic Multifocal Pancreatic Adenocarcinoma
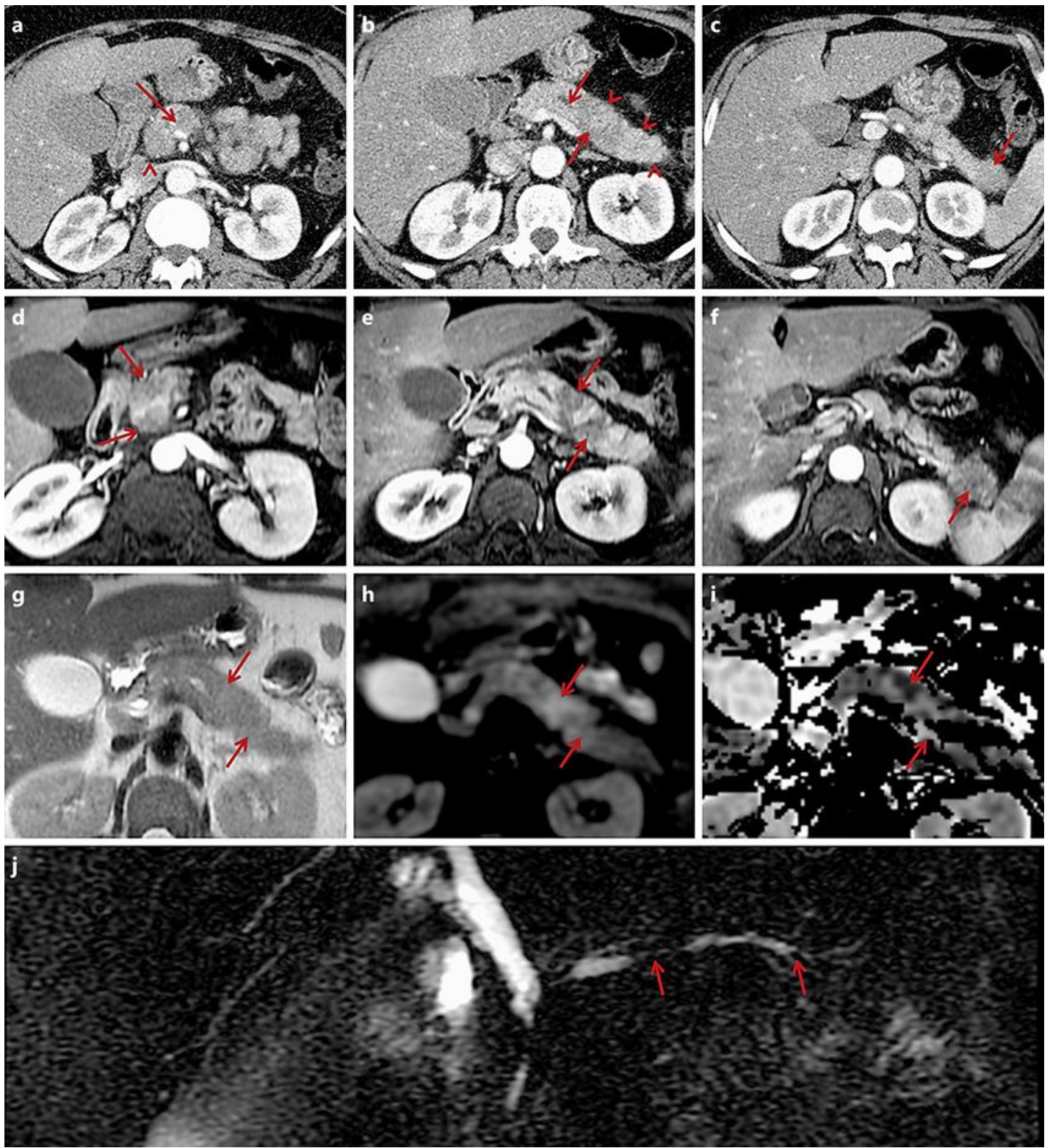
Fig. 1. Axial contrast-enhanced CT images of the pancreas in the arterial phase of imaging demonstrate multifocal hypoenhancing lesions (arrows) throughout the pancreatic head and neck (a), pancreatic body (b), and pancreatic tail (c). There is superimposed mild peripancreatic fat stranding (arrowheads) in keeping with inflammation and/or fibrosis. Axial contrast enhanced T1-weighted MR images of the pancreas in the arterial phase of imaging demonstrate multifocal hypoenhancing lesions (arrows) throughout the pancreatic head and neck (d), pancreatic body (e), and pancreatic tail (f). Corresponding axial T2-weighted MR image (g) shows that the lesions demonstrate corresponding isointense to slightly hyperintense signal. These lesions demonstrate hyperintensity on diffusion weighted images $(\mathbf{h})$ and hypointensity on the corresponding ADC map (i) reflecting restricted diffusion. Noncontrast heavily T2-weighted MRCP image of the pancreas in the coronal projection (j) demonstrates mild segmental stenoses of the main pancreatic duct (arrows) with minimal upstream dilatation.
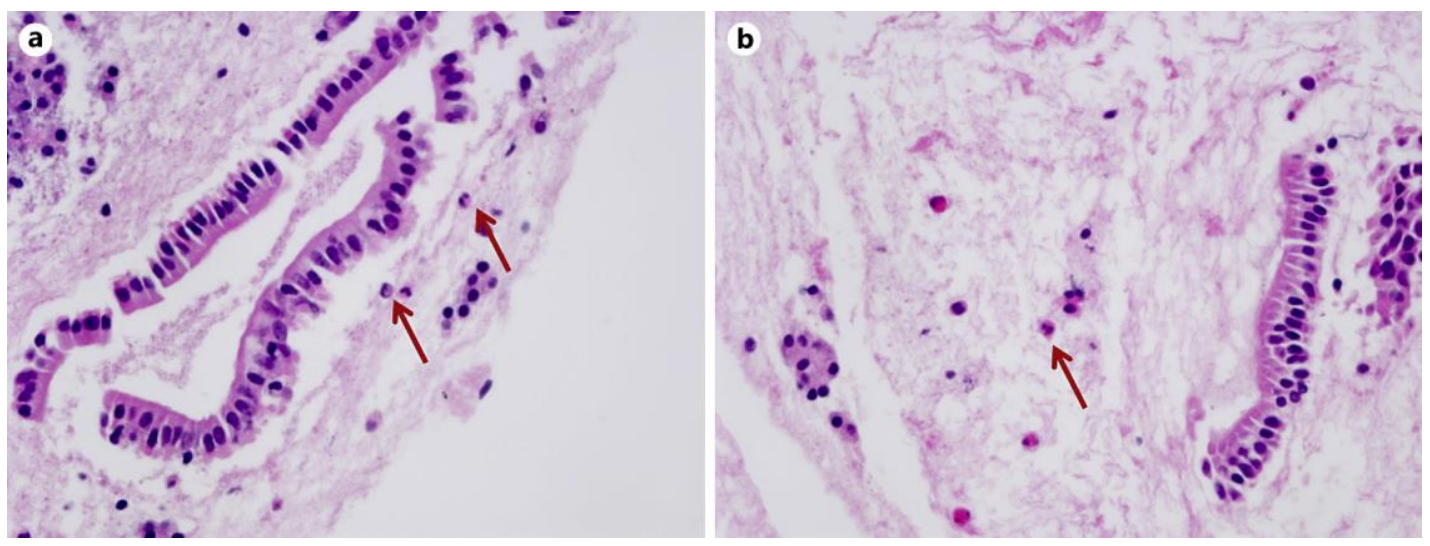

Fig. 2. Hematoxylin-eosin staining of fine needle aspiration specimens of the pancreatic mass-like lesions demonstrates a few neutrophils (arrows) in close approximation to the ductal epithelium suggestive of early granulocyte epithelial lesion formation.
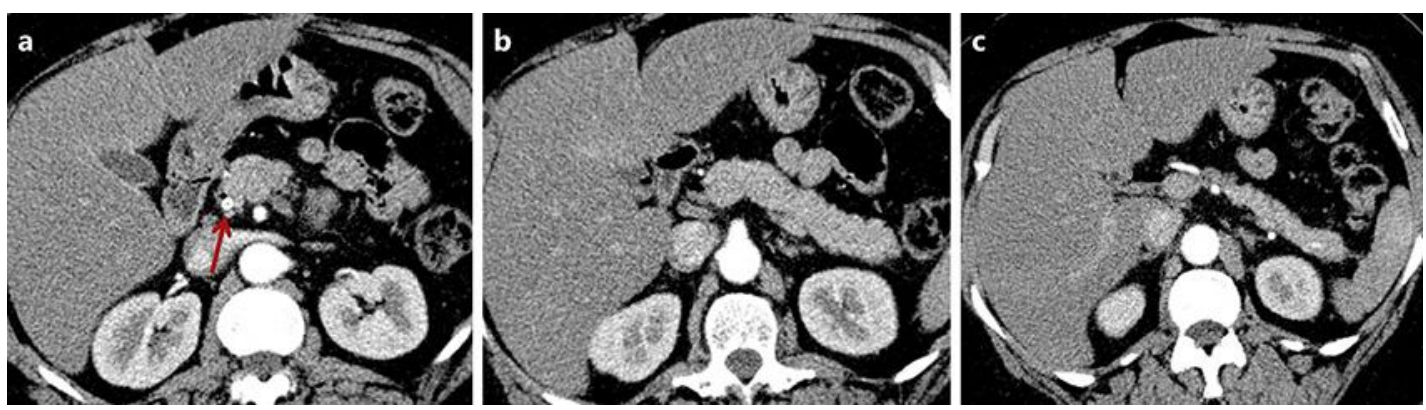

Fig. 3. One month following initiation of steroid treatment, axial contrast enhanced CT images (a-c) of the pancreas in the arterial phase of imaging demonstrate resolution of the previously seen multifocal lesions and peripancreatic stranding with normal homogenous enhancement of the pancreas. A stent in the common bile duct (arrow) was placed during the patient's initial hospitalization for a short segment stricture. 
Table 1. Patient's laboratory data on admission demonstrates elevated liver function tests with normal total IgG and marginally elevated IgG4

\begin{tabular}{lcc}
\hline Variable & Value & Normal range \\
\hline Total bilirubin, mg/dL & 1.7 & $0.2-1.0$ \\
Direct bilirubin, mg/dL & 0.8 & $0.0-0.3$ \\
Alkaline phosphatase, U/L & 265 & $35-125$ \\
Alanine aminotransferase, U/L & 246 & $14-54$ \\
Aspartate aminotransferase, U/L & 118 & $15-41$ \\
Lipase, U/L & 48 & $22-51$ \\
Total IgG, mg/dL & 1,140 & $565-1,765$ \\
IgG4, mg/dL & 90 & $4.0-86.0$ \\
CA 19-9, U/mL & $<1$ & $0-60$ \\
\hline
\end{tabular}

Table 2. Demographics, disease associations, imaging and histopathological features of autoimmune pancreatitis subtypes

\begin{tabular}{|c|c|c|}
\hline AIP Subtype & Type 1 & Type 2 \\
\hline Geographic prevalence & Asia >USA, Europe & Europe $>$ USA $>$ Asia \\
\hline Age & Older patients & Younger patients \\
\hline Gender & $\mathrm{M}>\mathrm{F}$ & $M=F$ \\
\hline Symptoms & Obstructive jaundice $>$ abdominal pain & Abdominal pain > obstructive jaundice \\
\hline $\begin{array}{l}\text { Extrapancreatic } \\
\text { pathology }\end{array}$ & $\begin{array}{l}\text { Xerostomia, xerophthalmia } \\
\text { Hilar lymphadenopathy } \\
\text { Sclerosing cholangitis } \\
\text { Interstitial nephritis } \\
\text { Retroperitoneal fibrosis }\end{array}$ & Ulcerative colitis \\
\hline Pancreatic imaging & $\begin{array}{l}\text { Diffuse enlargement >segmental masses } \\
>\text { multifocal masses }\end{array}$ & $\begin{array}{l}\text { Diffuse enlargement >segmental masses } \\
>\text { multifocal masses }\end{array}$ \\
\hline Serology & Elevated serum IgG4 & Normal serum IgG4 \\
\hline \multirow[t]{2}{*}{ Pathology } & $\begin{array}{l}\text { IgG4 plasma cells ( }>10 \text { cells/HPF) } \\
\text { Lymphoplasmocytic infiltrate } \\
\text { Storiform fibrosis }\end{array}$ & $\begin{array}{l}\text { None or few IgG4 plasma cells } \\
(<10 \text { cells/HPF })\end{array}$ \\
\hline & Vasculitis & Granulocyte epithelial lesions \\
\hline Steroid & Responsive & Responsive \\
\hline Relapse & Common & Uncommon \\
\hline
\end{tabular}

